\title{
CUESTIONARIO DE AFRONTAMIENTO DEL ESTRÉS (CAE): VALIDACIÓN EN UNA MUESTRA MEXICANA
}

\author{
MÓNICA T. GONZÁLEZ Y RENÉ LANDERO \\ Facultad de Psicología, Universidad Autónoma de Nuevo León, Monterrey, México
}

\begin{abstract}
Resumen: El artículo describe los resultados de la aplicación del Cuestionario de Afrontamiento del Estrés (Sandín, B. y Chorot, P. (2003). Cuestionario de Afrontamiento del Estrés (CAE): Desarrollo y validación preliminar. Revista de Psicopatología y Psicología Clínica, 8, 39-54), en una muestra de estudiantes de México $(n=365)$; con los objetivos de valorar su consistencia interna y estructura factorial; evaluar las correlaciones de afrontamiento con estrés percibido y síntomas somáticos, y una reducción del CAE. Los resultados confirman las 7 formas de afrontamiento y 2 factores de orden superior. Una diferencia con el estudio original fue la inclusión de religión en el factor de afrontamiento racional. Se confirmó la estructura factorial mediante análisis factorial confirmatorio (AFC). Se encontraron correlaciones entre algunas formas de afrontamiento, estrés percibido y síntomas. Por último, la reducción de la escala a 21 ítems mostró mejor ajuste en el AFC y adecuada consistencia interna.
\end{abstract}

Palabras clave: Afrontamiento, CAE, estrés, evaluación, análisis factorial.

\section{The Coping Stress Questionnaire (CAE): Validation in a Mexican sample}

\begin{abstract}
This paper describes the results of applying the Coping Stress Questionnaire [Cuestionario de Afrontamiento del Estrés, CAE] [Sandín, B. \& Chorot, P. (2003). Cuestionario de Afrontamiento del Estrés (CAE): Desarrollo y validación preliminar. Revista de Psicopatología y Psicología Clínica, 8, 39-54] in a Mexican students sample $(N=365)$, with the aim to verify its reliability and factor structure, to assess the correlation between coping, perceived stress and somatic symptoms, and the reduction of the CAE length. The seven dimensions of coping and the 2 higher order factors were confirmed, though a difference with the original study was found in the inclusion of religion in the rational focused coping factor. This factor structure was confirmed by confirmatory factor analysis (CFA). Correlations between some coping styles, perceived stress and some symptoms were found. Finally, a reduction of the questionnaire to 21 -items showed the best fit in the CFA and an adequate reliability.
\end{abstract}

Keywords: Coping, CAE, stress, assessment, factor analysis.

\section{INTRODUCCIÓN}

En términos generales el concepto de afrontamiento hace referencia a esfuerzos cognitivos y conductuales, que lleva a cabo el individuo para hacer frente al estrés (Sandín, 1995). El estrés psicológico se define como una relación particular entre el individuo y su entorno que es evaluado por el propio sujeto como amena-

Recibido: 5 enero 2007; aceptado: 25 septiembre 2007.

Correspondencia: M.C. Mónica Teresa González Ramírez, Cuerpo académico en Psicología Social de la Facultad de Psicología, Universidad Autónoma de Nuevo León, Mutualismo 110, Col. Mitras Centro, 64460 Monterrey, N.L., México. Correo e: monygzz77@yahoo.com zante o desbordante de sus recursos y, que pone en peligro su bienestar (Lazarus y Folkman, 1984), en esta definición de estrés se incluye la evaluación y el afrontamiento, que median la frecuencia, intensidad, duración y tipo de las respuestas psicológicas y somáticas; en este modelo se enfatiza el papel activo del individuo ante los procesos de estrés. En este sentido el sujeto no solo decide que es estresante, mediante ciertos procesos; sino que además, una vez asumida la fuente de estrés personal, puede transformarla mediante estrategias de afrontamiento (Peñacoba y Moreno, 1999).

Así, el individuo utiliza diversas formas de afrontamiento para tratar con el estrés, algunas 
pueden ser adaptativas, mientras que otras no lo son (Sandín y Chorot, 2003).

En las investigaciones en torno al afrontamiento se ha detectado una correlación entre algunas formas de afrontamiento y síntomas somáticos. Ejemplo de esto es la investigación realizada en Rusia por Ruchkin, Eisemann y Hägglöf (2000) quienes estudiaron a 185 jóvenes delincuentes y 86 controles, encontrando correlaciones significativas entre algunas formas de afrontamiento y síntomas somáticos. Algunas de ellas fueron: evitación cognitiva y síntomas de resfriado ( $r=0,20$ en controles); pedir ayuda y síntomas de gripe $(r=0,17)$; evitación cognitiva y síntomas de gripe $(r=0,21$ en delincuentes); evitación cognitiva con problemas circulatorios $(r=0,19)$; evitación conductual con malestares gástricos $(r=0,15)$; evitación conductual con síntomas de resfriado $(r=0,30)$, evitación conductual con fatiga $(r=0,26)$; evitación conductual con problemas de circulación $(r=0,32)$.

Otras investigaciones, como la realizada en España por Matud y Bethencourt (2000), evidencian la correlación entre síntomas somáti$\cos$ y afrontamiento emocional $(r=0,39)$, en este caso en mujeres con edad promedio de 46,6 años, encontrando correlación significativa entre síntomas y estilo de afrontamiento emocional. También en España, Viñas y Caparós (2000) encontraron que los estudiantes que utilizan estrategias paliativas (como es el afrontamiento centrado en las emociones, o el escape conductual o cognitivo), manifiestan un mayor malestar físico; encontraron correlaciones significativas entre escape cognitivo y síntomas respiratorios $(r=0,32)$, de alergia $(r=$ $0,25)$ y neurosensoriales $(r=0,22)$, así como, entre las formas de afrontamiento: centrarse en las emociones y búsqueda de apoyo social, con todos los factores (desde $r=0,23$ en síntomas cardiovasculares, hasta $r=0,33$ en síntomas del aparato genito-urinario). Por último, Sandín y Chorot (2003) indican que se ha encontrado correlación positiva de los síntomas con evitación, aceptación resignada y descarga emocional; y correlación negativa con solución de problemas y análisis lógico.

La evaluación del afrontamiento es clave, así, la decisión sobre el cuestionario a utilizar debe tomar en cuenta las propiedades psicométricas del mismo. Existen numerosos instrumentos para su evaluación (Olmedo, Ibáñez y Hernández, 2001); tal vez uno de los más utilizados ha sido el Ways of Coping Questionnaire (WOCQ) diseñado por Folkman y Lazarus, sin embargo, las investigaciones empíricas ponen de manifiesto su falta de validez y estabilidad de la estructura factorial (Olmedo et al., 2001). Así mismo, la teoría de Lazarus y Folkman ha recibido criticas (Pelechado, 2005).

Otra opción para la evaluación del afrontamiento es el Cuestionario de Afrontamiento del Estrés (CAE) de Sandín y Chorot (2003), que consta de 42 ítems y evalúa 7 formas de afrontamiento, comprobados a través de un análisis factorial con 592 sujetos (focalizado en la solución del problema, autofocalización negativa, reevaluación positiva, expresión emocional abierta, evitación, búsqueda de apoyo social, y religión); las 7 formas de afrontamiento se agrupan en 2 factores de orden superior, el primero corresponde a una dimensión relacionada fundamentalmente con el problema o la situación estresante y con componentes racionales, en tanto que el segundo factor representa el componente emocional del afrontamiento (Sandín y Chorot, 2003).

Las dos dimensiones identificadas en el CAE son las planteadas por Lazarus y Folkman (1989). El afrontamiento centrado en el problema se refiere a los esfuerzos dirigidos fundamentalmente a la fuente de origen del estrés para modificarla o eliminarla y buscar una solución satisfactoria para el sujeto. Por otro lado, el afrontamiento centrado en la emoción se refiere a los esfuerzos encaminados a regular los estados emocionales que están en relación con, o son una consecuencia de las situaciones estresantes (Lazarus y Folkman, 1989); en este caso el sujeto no modifica la situación, por lo que, en algunas ocasiones el manejo no es funcional (Casado, 2002).

Entre las ventajas del CAE consideramos sus propiedades psicométricas, el número de ítems y que fue diseñado en español. Considerando lo anterior, es de interés continuar la investigación en torno al CAE, buscando contar con mas evidencias de su fiabilidad y validez. Así, el presente estudio se enfoca a valorar la fiabili- 
dad de consistencia interna y la estructura factorial del CAE en una muestra de estudiantes de Psicología de una Universidad del Norte de México; así mismo, se evalúan las correlaciones entre las formas de afrontamiento, el estrés percibido y los síntomas somáticos, así como también una posible reducción del número de items del CAE.

\section{MÉTODO}

\section{Participantes}

Los participantes en el estudio fueron 365 estudiantes de psicología de una Universidad del Norte de México, de una población de 2,410 , seleccionados aleatoriamente, considerando la distribución por sexo y turno. La muestra se distribuye de la siguiente manera, $20,5 \%$ son hombres y $79,5 \%$ son mujeres. La edad promedio de los sujetos fue de 20,48 años $(D . T .=3,62)$, con una mediana de 20 años.

\section{Instrumentos}

Cuestionario de afrontamiento del estrés (CAE; Sandín y Chorot, 2003). Sus 7 dimensiones de afrontamiento han reflejado bajas correlaciones entre sí (Sandín y Chorot, 2003), lo que apoya la posibilidad de evaluar los estilos de afrontamiento de manera independiente. La fiabilidad medida por el alfa de Cronbach osciló entre 0,64 y 0,92 en los 7 factores.

Patient Health Questionnaire (PHQ; Kroenke, Spitzer y Williams (2002). Fue diseñado como una medida de la intensidad o severidad de los síntomas somáticos. Se compone de 15 ítems tipo Likert, con 3 opciones de respuesta. El rango de la escala es de 0 a 30 puntos. En el estudio de Kroenke et al. (2002) la consistencia interna fue de 0,80 . Interian, Allen, Gara, Escobar y Díaz-Martínez (2002) obtuvieron un alfa de 0,79 en una muestra que incluía hispanos y no hispanos $(N=172)$. Sin embargo, respecto a la validez; en la muestra de hispanos el PHQ tuvo una relación menor con los síntomas médicamente inexplicables y mayor con el dies- trés. Sobre aplicaciones realizadas en México, se cuenta con información de consistencia interna ( $\alpha=0,77$; González y Landero, 2006), pero no se han encontrado datos sobre validación del instrumento en México.

Escala de Estrés Percibido (PSS; Cohen, Kamarak y Mermelstein, 1983). Consta de 14 ítems con puntuación de nunca (0) a muy a menudo (4). Se aplicó la adaptación para México realizada por González y Landero (2007), que confirma la estructura factorial reportada por Cohen y Williamson (1988) para la versión original y cuya consistencia interna fue de $\alpha=$ 0,83 .

\section{Procedimiento de análisis estadístico}

Se siguió el procedimiento practicado por los autores del CAE, realizando un análisis factorial exploratorio (AFE) con el método de componentes principales (PCA) y rotación oblimín. Posteriormente se realizó un análisis factorial de segundo orden con las puntuaciones factoriales del primer análisis factorial exploratorio. Las correlaciones con estrés percibido y síntomas somáticos se realizaron mediante la correlación de Spearman debido a que los datos no siguieron una distribución normal; razón por la cual, las pruebas de diferencia de grupos, para comparar varones y mujeres se realizaron con la U de Mann Whitney. Estos análisis se realizaron con el SPSS para Windows. Asimismo, se realizó un análisis factorial confirmatorio mediante ecuaciones estructurales, utilizando el AMOS 5.0, con el método de máxima verosimilitud. La bondad del ajuste del modelo de análisis factorial confirmatorio se evaluó a través del estadístico $\chi^{2}$, la razón entre $\chi^{2}$ y el número de grados de libertad $\left(\chi^{2} / \mathrm{gl}\right)$, cuyo valor debe ser menor de 3 para indicar un ajuste adecuado; los índices Goodness of fit index (GFI), Adjusted goodness of fit index (AGFI), Normed fit index (NFI), The Tucker-Lewis coefficient (TLI), Comparative fit index (CFI) e Incremental fit index (IFI), cuyos valores deben ser cercanos a 1; y los índices Root mean square residual (RMR) y Root mean square error of approximation (RMSEA), cuyos valores deben ser menores de 0,05 . 


\section{RESULTADOS}

\section{Análisis factorial exploratorio}

Para la primera etapa del AFE el valor correspondiente a la prueba de Kaiser-MeyerOlkin $(=0,85)$ fue aceptable y la prueba de esfericidad fue significativa $\left(\chi^{2}=6578,335 ; g l\right.$ $=861 ; p<0,001)$. El número de factores sugerido por el método MAP (minimum average plaartial) fue de 3 , y de 9 factores por el criterio de autovalor mayor a $1(60,89 \%$ de varianza explicada), el gráfico de sedimentación sugiere 7 factores, que son los indicados por los autores del cuestionario.

Como se pretendía confirmar la estructura factorial presentada por Sandín y Chorot (2003), se realizó el AFE restringiendo a 7 factores, encontrando que la mayoría de los ítems se ajustaban a la estructura informada por sus autores; solamente los ítems 22, 24, 33, 39 y 40 presentaron diferencias. El ítem 22 saturó en el factor correspondiente a búsqueda de apoyo social $(0,408)$ y en el factor de solución de problemas $(0,33)$, en el estudio de Sandín y Chorot (2003) éste ítem también saturó en una dimensión adicional. Los ítems 24 y 33 presentaron pesos factoriales menores a 0,40 en todos los factores. El ítem 39 saturó en búsqueda de apoyo social $(0,59)$ en lugar de en el factor de expresión emocional $(0,07)$. Y el ítem 40 carga en autofocalización negativa $(0,408)$, además de en evitación que es donde corresponde según el estudio original $(-0,45)$. En la Tabla 1 se presentan las cargas factoriales de los ítems en las dimensiones señaladas por Sandín y Chorot (2003). Las correlaciones entre los 7 factores fueron bajas (de 0,01 a-0,25), al igual que en el estudio de los autores de la escala. Con lo anterior se confirman los factores, con leves diferencias en los ítems agrupados en cada factor.

Tabla 1. Cargas factoriales de los ítems del CAE* obtenidas a través del análisis de componentes principales y rotación oblimin

Cargas factoriales

Búsqueda de apoyo social (BAS) $(\alpha=0,91)$

6. Le conté a familiares o amigos cómo me sentía

0,77

13. pedí consejo a algún pariente o amigo para afrontar mejor el problema

0,79

20. Pedí a parientes o amigos que me ayudaran a pensar acerca del problema (r)

0,85

27. Hablé con amigos o familiares para que me tranquilizaran cuando me encontraba mal

0,79

34. Pedí a algún amigo o familiar que me indicara cuál sería el mejor camino a seguir (r)

0,82

41. Procuré que algún familiar o amigo me escuchara cuando necesité manifestar mis sentimientos (r)

0,88

Expresión emocional abierta (EEA) $(\alpha=0,79)$

4. Descargué mi mal humor con los demás

0,75

11. Insulté a ciertas personas

0,76

18. Me comporté de forma hostil con los demás (r)

0,79

25. Agredí a algunas personas (r)

0,84

32.Me irrité con alguna gente (r)

0,85

39. Luché y me desahogué expresando mis sentimientos

0,07

Religión (RLG) $(\alpha=0,87)$

7. Asistí a la Iglesia (r)

$-0,82$

14. Pedí ayuda espiritual a algún religioso (sacerdote $u$ otro)

21. Acudí a la Iglesia para rogar que se solucionase el problema (r)

$-0,64$

$-0,86$

28. Tuve fe en que Dios remediaría la situación

$-0,65$

35. Recé

$-0,77$

42. Acudí a la Iglesia a poner velas o rezar (r)

$-0,81$

Focalizado en la solución del problema (FSP) $(\alpha=0,83)$ 
Tabla 1. Cargas factoriales de los ítems del CAE* obtenidas a través del análisis de componentes principales y rotación oblimin (continuación)

\begin{tabular}{|c|c|}
\hline & Cargas factoriales \\
\hline 1. Traté de analizar las causas del problema para poder hacerle frente & 0,62 \\
\hline 8. Traté de solucionar el problema siguiendo unos pasos bien pensados & 0,71 \\
\hline 15. Establecí un plan de acción y procuré llevarlo a cabo (r) & 0,83 \\
\hline \multicolumn{2}{|l|}{ 22. Hablé con las personas implicadas para encontrar una solución al problema } \\
\hline 29. Hice frente al problema poniendo en marcha varias soluciones concretas (r) & 0,748 \\
\hline 36. Pensé detenidamente los pasos a seguir para enfrentarme al problema (r) & 0,76 \\
\hline \multicolumn{2}{|l|}{ Evitación $(E V T)(\alpha=0,68)$} \\
\hline 5. Cuando me venía a la cabeza el problema, trataba de concentrarme en otras cosas (r) & $-0,80$ \\
\hline 12. Me concentré en el trabajo o en otra actividad para olvidarme del problema (r) & $-0,78$ \\
\hline 19. Salí al cine, a cenar, a «dar la vuelta», etc., para olvidarme del problema & $-0,55$ \\
\hline 26. Procuré no pensar en el problema (r) & $-0,73$ \\
\hline 33. Practiqué algún deporte para olvidarme del problema & $-0,06$ \\
\hline 40. Intenté olvidarme de todo & $-0,45$ \\
\hline \multicolumn{2}{|l|}{ Autofocalización negativa $(A F N)(\alpha=0,66)$} \\
\hline 2. Me convencí de que hiciera lo que hiciera las cosas siempre me saldrían mal (r) & 0,618 \\
\hline 9. No hice nada concreto puesto que las cosas suelen ser malas & 0,48 \\
\hline 16. Comprendí que yo fui el principal causante del problema & 0,55 \\
\hline 23. Me sentí indefenso(a) e incapaz de hacer algo positivo para cambiar la situación (r) & 0,57 \\
\hline 30. Me di cuenta de que por mí mismo no podía hacer nada para resolver el problema (r) & 0,63 \\
\hline 37. Me resigné a aceptar las cosas como eran & 0,59 \\
\hline \multicolumn{2}{|l|}{ Reevaluación positiva $(R E P)(\alpha=0,67)$} \\
\hline 3. Intenté centrarme en los aspectos positivos del problema (r) & 0,52 \\
\hline 10. Intenté sacar algo positivo del problema (r) & 0,50 \\
\hline 17. Descubrí que en la vida hay cosas buenas y gente que se preocupa por los demás & 0,52 \\
\hline 24. Comprendí que otras cosas, diferentes del problema, eran para mí mas importantes & 0,20 \\
\hline 31. Experimenté personalmente eso de que «no hay mal que por bien no venga» (r) & 0,59 \\
\hline 38. Comprobé que, después de todo, las cosas podían haber ocurrido peor & 0,62 \\
\hline
\end{tabular}

* Los ítems están ubicados en los factores señalados por Sandín y Chorot (2003)

(r) Ítems que forman la versión reducida

\section{Análisis factorial de segundo orden}

Siguiendo el procedimiento empleado por Sandín y Chorot (2003) en una segunda etapa, se realizó en análisis factorial de segundo orden con las puntuaciones factoriales de la primera etapa, en el que encontraron 2 factores de orden superior (véase la Tabla 2). En ésta muestra se encontró que los dos factores explican el $43,63 \%$ de la varianza, levemente menor a lo encontrado por Sandín y Chorot $(49,3 \%)$, al igual que en su estudio, los autovalores de los factores restantes fueron menores a la unidad. Encontramos diferencias con los resultados de Sandín y Chorot en la correlación entre factores, los autores reportan una correlación de 0,20, mientras que en ésta muestra la correlación fue de 0,02. Asimismo, la distribución de las formas de afrontamiento en los factores de segundo orden muestra una diferencia, ya que religión se ubica en éste estudio en el factor de afrontamiento racional, mientras que en el estudio de Sandín y Chorot se ubica en el factor de afrontamiento emocional con una saturación de $-0,43$. Los pesos factoriales son en su mayoría muy similares a los encontrados por los autores, con excepción de los pesos para focalizado en la solución del problema, que en el estudio original tuvo un valor de 0,84 y de autofocalización negativa de 0,80 
Tabla 2. Análisis de componentes principales de orden superior para el CAE

\begin{tabular}{lcc}
\hline & \multicolumn{2}{c}{ Factores } \\
\cline { 2 - 3 } & Afrontamiento racional & Afrontamiento emocional \\
\hline Búsqueda de apoyo social & $\mathbf{0 , 7 3}$ & 0,14 \\
Expresión emocional abierta & $-0,10$ & $\mathbf{0 , 7 3}$ \\
Religión & $-\mathbf{0 , 4 7}$ & $-0,17$ \\
Focalizado en la solución del problema & $\mathbf{0 , 5 5}$ & $-0,31$ \\
Evitación & $-0,29$ & $\mathbf{0 , 5 6}$ \\
Autofocalización negativa & $-0,06$ & $\mathbf{0 , 6 5}$ \\
Reevaluación positiva & $\mathbf{0 , 6 2}$ & $-0,13$ \\
\hline
\end{tabular}

Nota. En negrita se indican las saturaciones elevadas, que fueron asignadas a cada factor.

\section{Análisis factorial confirmatorio}

Para el AFC se buscó en un primer momento valorar la estructura factorial informada por Sandín y Chorot (2003), para después valorar la inclusión de religión en el factor de afrontamiento racional, tal como lo indicó el análisis factorial de segundo orden previamente realizado. Así, en el primer análisis no se confirma la distribución de los factores de primer orden en los factores de segundo orden indicados por Sandín y Chorot; el peso de regresión de religión en el factor 2 no fue significativo. Se procedió a verificar la inclusión de religión en el factor 1 (afrontamiento racional), encontrando que el peso de regresión de religión en el factor 1 era significativo $(p<0,01)$. En ambos casos, no todos los parámetros fueron significativos (ítems 33 y 39). Así, se realizó un segundo modelo, donde se incluyeron en el factor 1: búsqueda de apoyo social, focalizado en la solución del problema, reevaluación positiva y religión (43,3\% de varianza estimada); y en el factor 2: expresión emocional abierta, autofocalización negativa y evitación $(40,7 \%$ de varianza estimada). Los indicadores de ajuste sugieren que el modelo 1 es susceptible de ser mejorado (Tabla 3 ).

Para mejorar el modelo y la medición de afrontamiento se fijaron a 0 los parámetros no significativos (ítems 39 y 33; en 2 momentos), mejorando levemente en el modelo 2 los estadísticos de bondad de ajuste (Tabla 3).

Por último, se valoró una reducción en la escala, disminuyendo a 3 indicadores en cada forma de afrontamiento, que es el mínimo requerido para cada factor común y permitir así la identificabilidad del modelo (Bollen, 1989; Fabrigar, MacCallum, Wegener y Strahan, 1999). Los ítems que se conservan fueron los que mostraron un peso factorial mayor en el AFC (en la Tabla 1 y Figura 1 puede observarse que ítems componen esta reducción del CAE). Esta reducción corresponde al modelo 3 (véa-

Tabla 3. Índices de bondad de ajuste de AFC

\begin{tabular}{|c|c|c|c|c|c|c|c|c|c|}
\hline & \multicolumn{5}{|c|}{ Índices de ajuste absoluto } & \multicolumn{4}{|c|}{ Índices de ajuste de incremento } \\
\hline & $\chi^{2} / \mathrm{gl}$ & GFI & AGFI & RMR & RMSEA & NFI & TLI & CFI & IFI \\
\hline Modelo 1 & 2,27 & 0,799 & 0,776 & 0,130 & 0,059 & 0,732 & 0,818 & 0,829 & 0,830 \\
\hline Modelo 2 & 2,12 & 0,817 & 0,795 & 0,121 & 0,056 & 0,763 & 0,849 & 0,858 & 0,859 \\
\hline Modelo 3 & 1,46 & 0,934 & 0,916 & 0,087 & 0,036 & 0,908 & 0,964 & 0,969 & 0,969 \\
\hline
\end{tabular}

Nota: GFI = Goodness of Fit Index (índice de bondad del ajuste), AGFI = Adjusted Goodness of Fit Index (índice de bondad de ajuste corregido), RMR = Root Mean Square Residual (residuo cuadrático medio), RMSEA = Root Mean Square Error of Approximation (error de aproximación cuadrático medio), NFI = Normed Fit Index (índice de ajuste normalizado), TLI $=$ Tucker-Lewis coefficient (coeficiente de Tucker y Lewis), CFI = Comparative Fit Index (índice de ajuste comparativo), IFI $=$ Incremental Fit Index (índice de ajuste incremental). 
se la Tabla 3), donde puede observarse que todos los estadísticos de bondad de ajuste son adecuados. La varianza estimada para el factor 1 es del $40 \%$ y de $60 \%$ para el factor 2 (Figura 1). Para facilitar la interpretación del modelo 3 (Figura 1) sólo se muestran los pesos de los ítems correspondientes a cada subescala, todos ellos presentan valores adecuados por lo que se consideran buenos indicadores de cada factor. Asimismo, se muestran los pesos de cada factor de primer orden (subescalas) en el factor de segundo orden correspondiente, siendo el valor más bajo el parámetro de la subescala de Religión, sin embargo, dado que todos los parámetros fueron significativos el modelo se considera adecuado. La correlación entre ambos factores sugiere que éstos forman parte de un mismo constructo (afrontamiento).

Para esta reducción del CAE se estimó nuevamente la consistencia interna obteniendo valores de alfa de 0,64 a 0,87 en las 7 formas de afrontamiento, y para los factores de segundo orden $\alpha=0,78$ para el factor 1 y $\alpha=0,71$ para el factor 2 .

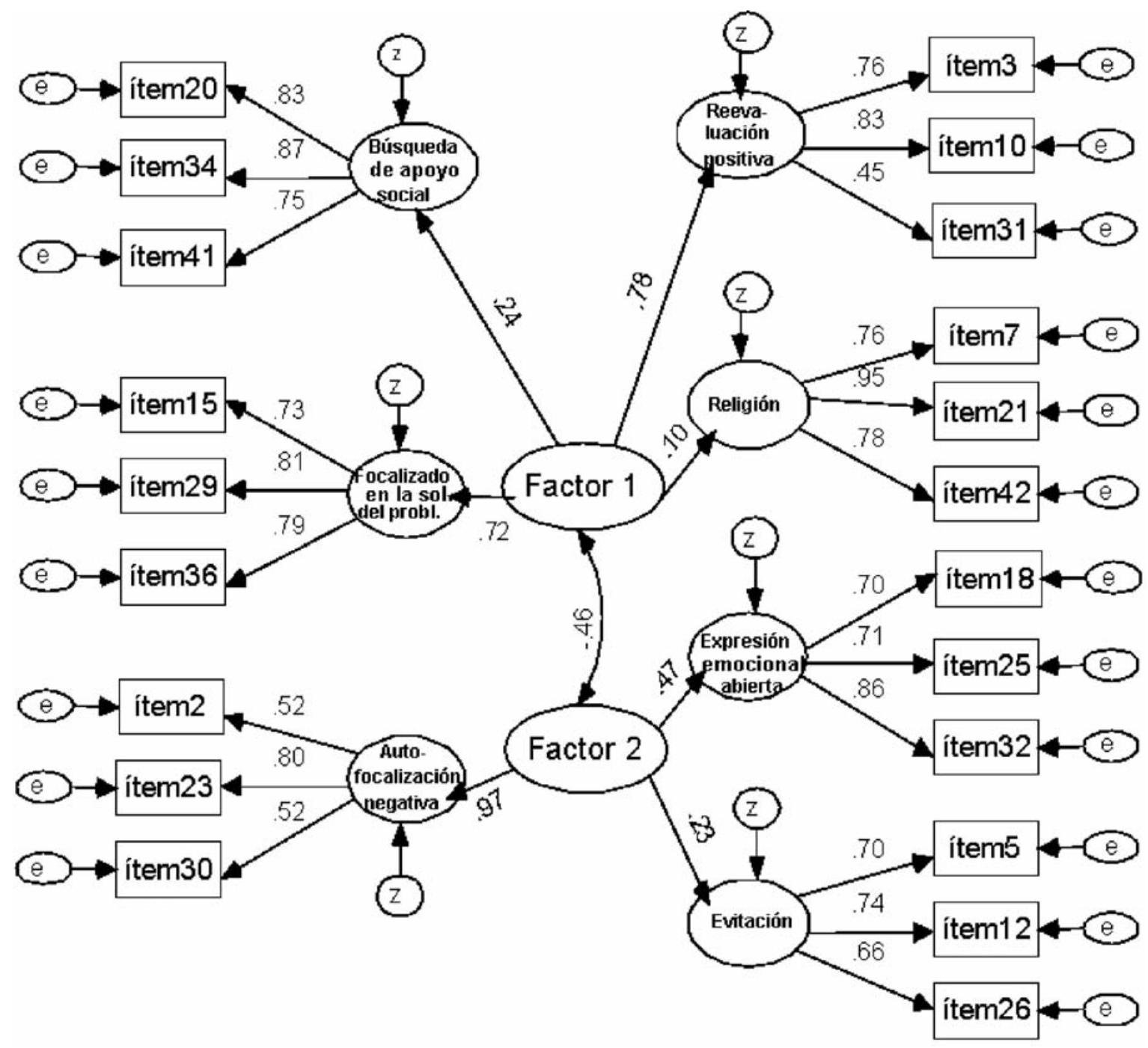

Figura 1. Representación gráfica del análisis factorial confirmatorio obtenido a partir de la versión reducida del CAE (modelo 3). En el modelo «e» y «Z» corresponden respectivamente a los errores de las variables exógenas y endógenas. 


\section{Diferencias por sexo y correlaciones}

Las puntuaciones medias, desviaciones típicas y medianas para las subescalas del CAE (cuestionario completo y abreviado), para estrés percibido y para síntomas se presentan en la Tabla 4. Cabe aclarar que se realizó la prueba de Kolmogorov-Smirnov, encontrando que los datos no se ajustaban a una distribución normal. Asimismo, se compararon todas las medidas de acuerdo al sexo, encontrando diferencias signi- ficativas para síntomas (PHQ) y para las formas de afrontamiento focalizado en la solución del problema, evitación, búsqueda de apoyo social y religión. En el estudio original (Sandín y Chorot, 2003) se informan diferencias similares, aunque se indica que en ninguna subescala los varones obtienen puntuaciones medias mayores que las mujeres; mientras que en este estudio los varones obtuvieron medias y medianas mas altas en la dimensión focalizado en la solución del problema (Tabla 4).

Tabla 4. Estadísticos descriptivos y prueba de normalidad

\begin{tabular}{|c|c|c|c|c|c|c|c|}
\hline \multirow[b]{2}{*}{ Variable } & \multicolumn{2}{|c|}{ Muestra total } & \multicolumn{2}{|c|}{ Varones } & \multicolumn{2}{|c|}{ Mujeres } & \multirow[b]{2}{*}{$Z^{1}$} \\
\hline & Mediana & Media $(D T)$ & Mediana & Media $(D T)$ & Mediana & Media $(D T)$ & \\
\hline PHQ & 6 & Media $(D T)$ & 5 & $5,65(3,71)$ & 6 & $7,09(4,11)$ & $-2,747 * *$ \\
\hline PSS & 21 & $6,79(4,07)$ & 20 & $20,56(6,18)$ & 22 & $22,29(7,20)$ & $-1,754$ \\
\hline CAE: & & $21,93(7,03)$ & & & & & \\
\hline Solución del problema & 16 & & 17 & $16,52(4,28)$ & 15 & $15,12(4,69)$ & $-2,440 *$ \\
\hline Autofocalización negativa & 5 & $15,41(4,64)$ & 6 & $5,96(3,67)$ & 5 & $5,62(3,39)$ & $-0,700$ \\
\hline Reevaluación positiva & 16 & $5,69(3,45)$ & 15 & $15,59(3,42)$ & 16 & $16,15(3,74)$ & $-1,338$ \\
\hline Expresión emocional $^{2}$ & 5 & $16,04(3,68)$ & 5 & $5,55(3,96)$ & 5 & $5,81(3,87)$ & $-0,483$ \\
\hline Evitación $^{2}$ & 10 & $5,75(3,89)$ & 8 & $8,33(3,71)$ & 10 & $9,90(3,80)$ & $-3,137 * *$ \\
\hline Búsqueda de apoyo social & 14 & $9,58(3,84)$ & 11 & $11,49(6,21)$ & 15 & $14,34(5,81)$ & $-3,730 * *$ \\
\hline Religión & 7 & $13,76(6,00)$ & 3 & $4,99(5,99)$ & 8 & $8,06(5,74)$ & $-4,829 * *$ \\
\hline CAE-Abreviado: & & $7,42(5,91)$ & & & & & \\
\hline Solución del problema & 7 & & 9 & $8,03(2,49)$ & 7 & $7,09(2,84)$ & $-2,630 * *$ \\
\hline Autofocalización negativa & 2 & $7,29(2,79)$ & 2 & $2,20(1,94)$ & 2 & $2,18(2,07)$ & $-0,382$ \\
\hline Reevaluación positiva & 9 & $2,18(2,04)$ & 9 & $8,13(2,29)$ & 9 & $8,32(2,32)$ & $-0,557$ \\
\hline Expresión emocional & 3 & $8,29(2,32)$ & 3 & $2,97(2,40)$ & 3 & $3,30(2,39)$ & $-1,234$ \\
\hline Evitación & 6 & $3,24(2,40)$ & 5 & $4,72(2,36)$ & 6 & $5,89(2,59)$ & $-3,623 * *$ \\
\hline Búsqueda de apoyo social & 7 & $5,65(2,56)$ & 5 & $5,35(3,14)$ & 7 & $6,80(3,10)$ & $-3,631 * *$ \\
\hline Religión & 2 & $6,50(3,16)$ & 0 & $1,89(3,14)$ & 3 & $3,58(3,27)$ & $-5,018 * *$ \\
\hline
\end{tabular}

${ }^{1}$ Valor estandarizado para la prueba $U$ de Mann-Whitney; ${ }^{2}$ Subescalas en las que se eliminó un ítem sobre la base del análisis factorial. ${ }^{*} p<0,05 ; * *<<0,01$.

Tabla 5. Correlaciones entre las dimensiones del CAE (entre paréntesis correlaciones de la versión abreviada), la sintomatología somática (PHQ) y el estrés percibido (PSS), y correlaciones entre las formas completa y abreviada del CAE

\begin{tabular}{lcccc}
\hline Dimensiones del CAE & \multicolumn{2}{c}{ PHQ } & \multicolumn{2}{c}{$\begin{array}{c}\text { Correlación entre } \\
\text { ambas formas del CAE }\end{array}$} \\
\hline Búsqueda de apoyo social (BAS) & $0,07(0,07)$ & 0,02 & $(0,04)$ & $0,96^{* *}$ \\
Expresión emocional abierta (EEA) & $0,19^{* *}\left(0,16^{*}\right)$ & $0,35^{* *}\left(0,33^{* *}\right)$ & $0,94^{* *}$ \\
Religión (RLG) & $0,13^{* *}(0,06)$ & 0,03 & $(0,01)$ & $0,89^{* *}$ \\
Focalizado en la solución del problema (FSP) & $-0,04(-0,05)$ & $-0,41^{*}\left(-0,39^{*}\right)$ & $0,94^{* *}$ \\
Evitación (EVT) & $0,11^{*}(0,08)$ & $0,17^{*}(-0,12)^{* *}$ & $0,88^{* *}$ \\
Autofocalización negativa (AFN) & $0,24^{* *}\left(0,26^{* *}\right)$ & $0,46^{* *}\left(0,57^{* *}\right)$ & $0,80^{* *}$ \\
Reevaluación positiva (REP) & $0,02 \quad(-0,01)$ & $-0,32^{* *}\left(-0,38^{* *}\right)$ & $0,84^{* *}$ \\
\hline
\end{tabular}

$* p<0,05 ; * * p=0,001$. 
Como último análisis se calcularon las correlaciones de cada una de las 7 dimensiones del CAE con el PSS y el PHQ (véase la Tabla 5). Éstos análisis se realizaron también con la versión reducida del CAE. Las formas de afrontamiento que correlacionan negativamente con estrés percibido son: focalización en la solución del problema y reevaluación positiva. Mientras que las formas de afrontamiento que correlacionan positivamente con estrés percibido son: autofocalización negativa, expresión emocional abierta y evitación. Búsqueda de apoyo social y religión no se relacionaron significativamente al estrés. Las formas de afrontamiento que presentan correlaciones positivas y significativas con los síntomas son autofocalización negativa, expresión emocional abierta, evitación y religión (las dos últimas solo en la versión original).

\section{DISCUSIÓN}

En la primera etapa del análisis factorial se confirmó la estructura factorial del CAE con leves diferencias a lo informado por Sandín y Chorot (2003) en la agrupación de ítems en cada factor.

En la segunda etapa se realizó un análisis factorial de segundo orden con las puntuaciones factoriales de la primera etapa; en esta etapa se encontró una diferencia con el estudio de Sandín y Chorot (2003). Los autores de la escala informaron 2 factores de orden superior; el primero representa la dimensión relacionada fundamentalmente con el problema o la situación estresante, y con componentes lógicos o racionales, éste primer factor se compone de los factores de búsqueda de apoyo social, focalizado en la solución del problema y reevaluación positiva; el segundo factor representa los componentes emocionales del afrontamiento y se compone de los factores de expresión emocional abierta, autofocalización negativa, evitación y religión (saturando éstas 2 últimas negativamente en el segundo factor de orden superior); la correlación que encontraron entre ambos factores fue de $-0,20$ y la varianza explicada fue de $29,30 \%$ para el factor 1 y de $20,01 \%$ para el factor 2. Las diferencias encontradas con los resultados de Sandín y Chorot son en la corre- lación entre los factores y en la ubicación de la forma de afrontamiento religión en el factor de afrontamiento racional, mientras que en el estudio de Sandín y Chorot se ubica en el factor de afrontamiento emocional; esta estructura factorial para los factores de orden superior se confirmó con el AFC. Los resultados sugieren que existen diferencias entre los estudiantes mexicanos y los españoles en cuanto a esta forma de afrontamiento al considerarla racional o emocional. Consideramos que estas diferencias pueden atribuirse a cuestiones culturales.

La reducción propuesta para el CAE, con solo 3 indicadores para cada forma de afrontamiento cuenta con valores de consistencia interna adecuados para cada subescala. El AFC realizado para esta versión mostró una mejoría en los estadísticos de bondad de ajuste en comparación con la versión completa. Presentando la ventaja de ser una versión abreviada con correlaciones adecuadas con la versión original, fiabilidad y validez aceptable.

En cuanto a las correlaciones con estrés percibido y síntomas somáticos como se mencionó previamente, las formas de afrontamiento relacionadas a bajos niveles de estrés percibido son: focalización en la solución del problema y reevaluación positiva; retomando los ítems de cada subescala, solución del problema se refiere a la acción directa y racional para solucionar las situaciones problemáticas, lo cual es congruente con la definición de Fernández-Abascal (1997) sobre esta forma de afrontamiento, y reevaluación positiva se refiere al afrontamiento enfocado a crear un nuevo significado (positivo) a la situación problema; ambas son formas activas de afrontamiento.

Las formas de afrontamiento relacionadas a altos niveles de estrés percibido son: autofocalización negativa, que es el lado opuesto a la reevaluación positiva, es decir, enfocarse a lo negativo de sí mismo, como responsable del problema; expresión emocional, que se refiere a manifestaciones expresivas hacia otras personas de la reacción emocional negativa, relacionada al problema (activa), y evitación, que se refiere a pensar o hacer otras cosas, ignorando el problema o situación estresante.

En búsqueda de apoyo social se agruparía lo que Fernández-Abascal (1997) considera apoyo 
social emocional y apoyo social al problema, que se refieren a la búsqueda de apoyo para la situación emocional en que se encuentra, además de buscar consejo en los demás para solucionar el problema. Para esta muestra, religión y búsqueda de apoyo social no son formas de afrontamiento que estén asociadas al estrés percibido.

En cuanto a la correlación con síntomas somáticos, se presentaron diferencias en la versión original y reducida de las subescalas de evitación y religión. En todos los casos las correlaciones son bajas; por lo que a pesar de ser significativas pudieran ser espurias o debidas a la muestra estudiada.

Asimismo, las correlaciones de cada subescala reducida con la subescala original correspondiente, son superiores a 0,80 y significativas. Así, consideramos que la reducción de la escala proporciona la ventaja en el tiempo de aplicación, sin perder información de cada forma de afrontamiento; por lo que recomendamos se continúe explorando sus propiedades psicométricas en otras muestras.

Las aportaciones del presente estudio son en primer lugar la validación del CAE en una muestra mexicana y en segundo lugar, la propuesta de una versión reducida. Contar con instrumentos validos y fiables es necesario para la investigación y la evaluación en terapia, así, el afrontamiento es un elemento central en el manejo del estrés, por lo que el uso del CAE es una buena opción tanto para terapia, como para investigaciones relacionadas al tema.

\section{REFERENCIAS BIBLIOGRÁFICAS}

Bollen, K.A. (1989). A new incremental fit index for general structural equation models. Sociological Methods and Research, 17, 303-316.

Casado, F. (2002). Modelo de afrontamiento de Lazarus como heurístico de las intervenciones psicoterapéuticas. Apuntes de Psicología, 20, 403-414.

Cohen, S., Kamarak, T., y Mermelstein, R. (1983). A Global Measure of Perceived Stress. Journal of health and social behaviour, 24, 385-396.

Cohen, S., y Williamson, G. (1988). Perceived stress in a probability sample of the U.S. In S. Spacapam \& S. Oskamp (Eds.), The social psychology of health: Claremont Symposium on Applied Social Psychology. Newbury Park, CA: Sage.
Fabrigar, L., MacCallum, R., Wegener, DT, y Strahan, EJ. (1999). Evaluating the use of Exploratory Factor Analysis in Psychological Research. Psychological Methods, 4, 272-299.

Fernández-Abascal, E. (1997). Estilos y estrategias de afrontamiento. En E. Fernández-Abascal, C. Palmer, M. Chóliz y F. Martínez (Eds.), Cuadernos y prácticas de motivación y emoción. España: Pirámide.

González, M.T., y Landero, R. (2006). Síntomas psicosomáticos y estrés en estudiantes de Psicología. Revista de Psicología Social, 21, 141-152.

González, M.T., y Landero, R. (2007). Factor Structure of the Perceived Stress Scale (PSS) in a Sample from Mexico. The Spanish Journal of Psychology, 10, 199206.

Kroenke K, Spitzer RL, y Williams, J. (2002). The PHQ15: Validity of a new measure for evaluating the severity of somatic symptoms. Psychosomatic Medicine, 64, 258-266.

Lazarus, R., y Folkman, S. (1984). Stress, coping and adaptation. New York: Springer.

Lazarus, R., y Folkman, S. (1989). Estrés y procesos cognitivos. Barcelona: Editorial Martínez Roca.

Matud, M., y Bethencourt, J. (2000). Ansiedad, depresión y síntomas psicosomáticos en una muestra de amas de casa. Revista Latinoamericana de Psicología, 32, 91106.

Olmedo, E., Ibáñez, I., y Hernández, L. (2001). Validación estructural del Ways of doping questionnaire de Lazarus y Folkman: un análisis factorial restrictivo. Ansiedad y estrés 7, 15-28.

Pelechado, V. (2005). Acerca del afrontamiento y enfermedad crónica: una reflexión ¿fundada? Revista de Psicopatología y Psicología Clínica, 10, 3-14.

Peñacoba, C., y Moreno, B. (1999). La escala de estresores universitarios (EEU). Una propuesta para la evaluación del estrés en grupos de poblaciones especificas. Ansiedad y estrés 5, 61-78.

Ruchkin, V., Eisemann, M., y Hägglöf, B. (2000). Coping styles and psychosomatic problems: Are they related? Psychopathology, 33, 235-239.

Sandín, B. (1995). El estrés. En A. Belloch, B. Sandín y F. Ramos (Eds.). Manual de psicopatología, Vol. 2 (pp. 3-52). Madrid: McGraw-Hill.

Sandín, B., y Chorot, P. (2003). Cuestionario de afrontamiento del estrés (CAE): Desarrollo y validación preliminar. Revista de Psicopatología y Psicología Clinica, 8, 39-54.

Viñas, F., y Caparrós, B. (2000). Afrontamiento del periodo de exámenes y sintomatología somática autoinformada en un grupo de estudiantes universitarios. Psicología.com, 4 (1) Revisado el 26 de marzo del 2002. Disponible en: http://www.psiquiatria.com/psicologia/vol4num1/ art_5.htm 